\title{
Инцидентность венозных тромбозов в европейской популяции: роль хирургических вмешательств
}

\begin{abstract}
В статье рассматрено инцидентность и роль хирургических вмешательств в развитии венозных тромбозов.
По данным проведенного обзора установлено, что свыше 70 \% тромбозов глубоких вен нижних конечностей после общехирургических операций протекают бессимптомно, при этом фатальная тромбэмболия легочной артерии часто является первым и единственным проявлением венозного тромбоза, занимая третье место в общей структуре причин внезапной смерти. Венозные тромбоэмболические осложнения сопровождаются поверхностным тромбофлебитом у 25 \% больных, при этом в большинстве случаев диагностируется тромбоз глубоких вен, а в 3,9 \% случае возникает тромбоэмболия легочной артерии. Среди факторов хирургического риска венозных тромбоэмболий отмечена связь с общей анестезией, длительностью операции более 2 часов, тромбоцитозом, наличием асцита, хронической сердечной недостаточностью, гипоальбуминемией.
\end{abstract}

Ключевые слова: венозный тромбоз; инцидентность; хирургические вмешательства.

Венозный тромбоз - острое заболевание, характеризующееся образованием тромба в просвете вены с выраженным или невыраженным воспалительным процессом и нарушением кровотока. Наличие воспаления стенки вены или (и) окружающих тканей в зоне тромбоза способствует другому названию этого заболевания - тромбофлебит (ТФ) [9].

Использование высококачественных эффективных технологий в оперативной хирургии, проведение высококачественной анестезиологической поддержки, своевременная, основанная на принципах доказательной медицины, передоперационная подготовка и активное послеоперационное ведение пациентов способствовали закономерному уменьшению встречаемости послеоперационных осложнений. На этом фоне немаловажную роль в развитии влияния на послеоперационную летальность - ближайшую и отдаленную - начинают играть такие осложнения, как тромбоз глубоких вен (ТГВ) нижних конечностей, тромбозы поверхностных вен (ТПВ) и тромбоэмболия легочной артерии (ТЭЛА), которые объединяются общим понятием “венозного тромбоэмболизма” (ВТЭ) [4]

Тромбоэмболические заболевания по-прежнему остаются одной из основных причин смертности и инвалидизации населения в индустриально развитых странах. Венозный тромбоз имеет мультифакторную природу: наряду с приобретенными факторами риска важную роль в его развитии играет генетическая предрасположенность [20,38].

Цель работы: проанализировать доступные данные, посвященные исследованию инцидент- ности венозных тромбоэмболий в абдоминальной хирургии, а также клинических особенностей данной патологии.

Клиническая структура венозных тромбоэмболий. Разные авторы указывают на частоту встречаемости венозных тромбозов в очень широких пределах, так частота их развития колеблется от 20 до $59 \%$ [5, 15]. При этом свыше $70 \%$ тромбозов глубоких вен нижних конечностей после общехирургических операций протекают бессимптомно и не диагностируются [11]. Фатальная тромбоэмболия легочной артерии нередко является первым и единственным проявлением венозного тромбоза и занимает третье место в общей структуре причин внезапной смерти [29].

Частота возникновения венозного тромбоза (ВТ) и тромбоэмболических осложнений в общей популяции составляет 1,0-2,0:1000 населения ежегодно $[18,39]$. Важной чертой ВТ является многофакторный характер его этиопатогенеза [23].

Венозные тромбоэмболические осложнения сопровождаются поверхностным тромбофлебитом (ТФ) у 25 \% больных. В большинстве случаев диагностируется тромбоз глубоких вен (ТГВ), а в 3,9 \% случаев возникает тромбоэмболия легочной артерии (ТЭЛА) [32]. Летальность в сроки от 3 до 6 мес. после эпизода ТГВ составляла, по данным авторов, от 7 до 15 \% [14, 35]. При этом установленными причинами смерти были рак, ТЭЛА и массивное кровотечение. ПТФ у 9,3 \% больных распространялся на глубокие магистральные вены, в 0,5-1 \% случае из них возникала ТЕЛА, в 95 \% инвалидизирующая посттромбофлебитическая болезнь (ПТХ) [8], при ко- 
торой, в свою очередь, риск возникновения ТЭЛА равнялся 20-45 \% [24].

Тромбоз в системе вен нижних конечностей и нижней полой вены является прогностически наиболее опасным видом данного патологического процесса. В структуре поражений от всех видов тромбозов частота поражения этой системы составляет до 95 \%. Ежегодно возникновение новых случаев венозного тромбоэмболизма (ТГВ + TEЛА) составляет от 80 до 120 больных на 100000 населения. До 30 из них умирают в течение 30 дней от момента диагноза, еще у 20 пациентов в течение 2 лет возникает рецидив заболевания.

Распространенность венозных тромбозов в европейской популяции. По данным Британского реестра заболеваний от 2015 года, количество смертей, связанных напрямую с развитием венозного тромбоэболизма (ВТЭ), составляет 25000 ежегодно, что превышает суммарный показатель смертности, в которую включены рак молочной железы, смертность от осложнений вируса иммунодефицита (ВИЧ) и смертность вследствие дорожных аварий [37].

Инцидентность венозного тромбоэмболизма, связанного непосредственно с оперативными вмешательствами, также весьма разнообразно. Установлено, что смертность от любого послеоперационного осложнения, включая и ВТЭ, зависит непосредственно от типа и вида операционного вмешательства [22]. По данным Hospital Episode Statistics (HES) [34], у больных с предшествующей диагностированной патологией вен (величина группы обследуемых составляла - 35374 пациентов) инцидентность ВТЭ составляла 0,51 \%. Таким образом, в послеоперационном интервале ВТЭ связанный с непосредственным поражением сосудов не играет столь важной роли, как предполагалось. Причем в этом же исследовании отмечена связь с увеличением частоты ВТЭ с применением общей анестезии.

В данном исследовании [31], при оценке только внутригоспитального ВТЭ, связанного и несвязанного с оперативными вмешательствами, установлен иной характер инцидентности ВТЭ, а именно 74,5 на 100 пациенто-лет. При этом основными предикторами ТГВ считали возраст, наличие неспецифического язвенного колита, опухоли любой локализации, предшествующий прием глюкокортикоидов и оральных контрацептивов. А ТЭЛА ассоциировалась с наличием ИБС, сердечной недостаточности и цереброваскулярными изменениями.

Данное исследование послеоперационного ВТЭ (всего 75771 пациентов) [30] установило также низкую инцидентность его развития - всего 0,68 \% случаев клинически выраженного ВТЭ. Правда, 30-дневная летальность в связи с ВТЭ была в 4 раза больше без наличия ВТЭ (16,9 \% vs 4,4 \%, p < 0001). Также в данном трайле отмечено отсутствие вариативного ежегодного тренда развития ВТЭ (от 0,65 до 0,72 \% в течение 5 лет наблюдений). В этом исследовании дооперационные предикторы ТГВ и ТЭЛА были установлены следующие - возраст, женский пол, наличие хронического обструктивного заболевания легких (ХОЗЛ), низкий уровень альбумина, снижение уровня гемоглобина. Послеоперационными предикторами были наличие пневмонии, инфекции мочеполовых путей, гемотрансфузии, перенесенный инфаркт миокарда, почечная недостаточность. Однако проведение гемодиализа и повышенный уровень альбумина были установлены в качестве протективных факторов при ВТЭ.

Очень важные результаты получены в популяционном исследовании 2015 г. проведенном на 168005 пациентов [25]. Исследование, в отличии от HES, свидетельствовало о том, что основная масса случаев ВТЭ (64 \%) возникает в течение 90 дней после операционного вмешательства. Кроме того, установлена общая заболеваемость ВТЭ, составляющая 23,7 на 1000 пациенто-лет, которая колебалась от 3,2 на 1000 пациенто-лет у больных с геморроидэктомией до 118,3 на 1000 пациентолет при оперциях в области гастроэзофагеального отдела. В клинической практике частота фиксации ВТЭ связана с ведением пациентов внутрибольнично, хотя и внутрибольничная ВТЭ и ВТЭ, возникшая после выписки, приводила к значимому увеличению 90-дневной послеоперационной летальности. Также в этом исследовании были определены прогностические факторы развития тромбоэмболизма в послеоперационном периоде, а именно неотложное хирургическое вмешательство $(\mathrm{OR}=1,9195$ \% CI 1,60 - 2,28, $\mathrm{p}<0,001)$, возраст OR = 1,02 95 \% CI 1,02-1,03, p<0,001), увеличение индекса массы тела OR = 1,03 95 \% CI 1,011,04, $<$ <0.001), предыдущий эпизод ВТЭ (OR = 8,07 95 \% CI 6,61-9,83, p<0,001), длительность нахождения больного в стационаре, а также наличие любого из опухолевого процесса от II до IV стадий.

В целом, выше описанное исследование подтвердило результаты раннего (1992 г.) исследования [33], в котором были установлены факторы риска ВТЭ, преимущественно связанными с операциями в тазовой полости, а именно 10-кратное возрастания риска смерти от ТЭЛА в связи с ТГВ при наличие более старшего возраста, неотложности операции, женского пола, увеличения массы тела. Так- 
же в этом исследовании указывается на позитивный эффект подкожных инъекций гепарина каждые 12 часов до полной мобильности пациента.

Роль хирургических вмешательств и онкологических заболеваний в развитии венозных тромбозов. Отдельное исследование было проведено в отношении развития ВТЭ у пациентов с онкологическими оперативными вмешательствами (2006 2008 гг., всего 159039 пациентов) [26]. Инцидентность, установленная для ВТЭ, равнялась 1,6 \% от всех пациентов, причем 4,2 \% для оперативных гастроэзофагеальных вмешательств и 3,6\% для гепатопанкреатобилиарной хирургии. Кроме таких предикторов, как возраст, пол, тип опухоли и тип вмешательства, для развития ВТЭ в этом исследовании значимыми факторами риска являлись длительность операции более 2 часов, тромбоцитоз, наличие асцита, хронической сердечной недостаточности, гипоальбуминемия (во всех случаях $\mathrm{p}<0,001)$.

Но в данном когортном исследовании развития ВТЭ (83 203 пациентов с операбельным раком и 577207 контролей) [21] было указано более значимые величины инцидентности по данному осложнению, а именно - 13,9 случая на 1000 человеко-лет, а при локализации процесса в панкреатодуоденальной зоне - 98 (ДИ = 80-119) случаев на 1000 человеко-лет. При этом в данном исследовании определен ежегодный тренд развития ТГВ и ТЭЛА относительно возраста пациента и основного диагноза.

Важное исследование [19] было посвящено развитию ВТЭ в зависимости от избирательности хирургического вмешательства (всего охвачено 76 процедур) и степени их ургентности (всего в исследование включено 1653275 пациента за период от января 1992 по июнь 1996 г.). Степень инцидентности первичного ТГВ равнялась 0,8 \%, (95 \% ДИ = 0,7-0,9 \%), при этом частота развития самого грозного осложнения - ТЭЛА наблюдалась чаще, чем в ранее представленных трайлах - 37 \%, 95 \% CI = 36-38 \%. Среди абдоминальных операций риск развития ТГВ в первую очередь ассоциировался при проведении холецистэктомии. Это исследование также определенно свидетельствует, что риск развития тромбоэмболического осложнения достоверно возрастает и в отстроченном послеоперационном периоде - а именно более 91 дня с момента операций.

Проведены клинические исследования, посвященные также и особенностям возникновения тромбоэмболизма в 30-дневный срок после амбулаторной хирургии с оценкой соответствующих факторов риска (ACS-NSQIP) [17]. В этом слу- чае инцидентность колебалась в зависимости от установленных риск-факторов от 0,06 до 1,18 \%. Данными факторами риска были активный рак (OR 3,66, p=0,005), возраст 41-59 (OR 1,72, $\mathrm{p}=0,008)$, возраст $\geq 60$ (OR 2,48, $\mathrm{p}<0,001)$, индекс массы тела $\geq 40$ (OR 1,81, p=0,015), длительность операции $\geq 120$ мин. (OR 1,69, p=0,027), артроскопическая хирургия (OR 5,16, p<0,001).

B урологической практике при исследовании рисков развития тромбоэмболий у 126891 пациента, подвергшихся разным видам оперативных вмешательств в течении 12-месячного наблюдения, 839 больных (0,66 \%) были заново госпитализированы с диагнозом ВТЭ. При этом у 373 из них (0,29 \%) был диагноз ТГВ, но 466 (0,37 \%) госпитализированы с ТЭЛА.

Связь тромбоэмболизма с полом и типом операционных вмешательств установлена в Million Women Study (Великобритания) [12]. Риск ВТЭ статистически значимо возрастал в первые 12 недель после операции в связи с госпитализацией пациентки. В среднем 1 из 140 женщин после госпитализации и операции была повторно госпитализирована в связи с ВТЭ в течении 12 недель (при абдоминальном раке - 1 на 85 женщин) в сравнении 1 на 815 женщин с минимальными (амбулаторными) хирургическими вмешательствами, и 1 на 6200 в течении 12-недельного периода при отсутствии какой-либо хирургической манипуляции.

По данным НИИ скорой помощи (2002), 36 \% больных с разлитым перитонитом погибают от тромбоза глубоких вен нижних конечностей, осложнившегося фатальной ТЭЛА. В раннем послеоперационном периоде у пациентов общехирургического профиля при помощи УЗДС, ТГВ выявлено у 11 \%, причем тромбоз возникал, несмотря на проводимую стандартную профилактику [2, 3].

При использовании теста с меченым фибриногеном тромбы в венозном русле нижних конечностей после операций на органах брюшной полости обнаруживали в 28-33 \%, а у онкологических больных до 66 \% случаев, но только у 0,73,5 \% возникают клинические проявления этого осложнения [1].

Роль венозных тромбозов в развитии тромбоэмболии легочной артерии. Внедрение в практику высокотехнологичных, малоинвазивных вмешательств не привела к ожидаемому снижению ВТО. При лапароскопических вмешательствах менее выражены изменения в системе гемостаза по сравнению с традиционными операциями. Однако при лапароскопических операциях из-за кар- 
боксиперитонеума отмечено выраженное снижение объемной и линейной скорости кровотока в нижней полой вене и венах нижних конечностей $[6,7]$.

В течение многих лет ТГВ нижних конечностей и ТЭЛА считались отдельными заболеваниями. В настоящее время достоверно установлено, что почти в 70 \% больных при подтвержденной ТЭЛА имеется ТГВ нижних конечностей [27], и почти в 50 \% - при ТГВ нижних и верхних конечностей наблюдается бессимптомное течение ТЭЛА [10, 36]. Тромбы в венах голени у госпитализированных больных обнаруживают достаточно часто [13]. Частота бессимптомного течения тромбоза вен голени значительно отличается в зависимости от профиля пациентов: в общей хирургии она представляет от 5 до 30 \%, после больших ортопедических операций - от 40 до 70 \% [28].

С клинической точки зрения, большее значение имеет острый ТФ подкожных вен нижних конечностей, поскольку именно при такой локализации процесс тромбообразования имеет склонность к распространению по глубокой венозной системе со всеми вытекающими последствиями: от развития ТЭЛА в острой стадии заболевания, до формирования ХВН в отдаленные сроки наблюдения. При активном использовании инструментальных методов исследования ТГВ обнаруживают не менее чем у 10 \% больных с наличием предшествующего ТФ. Во многих ситуациях развитие тромбоза глубоких вен можно предупредить. Если это своевременно не сделать, патологический процесс трансформируется в принципиально другое состояние. Даже если у больного и не возникнет ТЭЛА, распространение тромбоза по магистральным венам и дальнейшее формирование ПТХ приведет к потребности

\section{СПИСОК ЛИТЕРАТУРЫ}

1. Затевахин И. И. Роль ультразвукового ангиосканирования в ранней диагностике бессимптомных острых венозных тромбозов системы нижней полой вены у оперированных больных / И. И. Затевахин // Материалы III Конференции ассоциации флебологов России. - Ростов-на-Дону, 2001. - С. 191.

2. Интеграл факторов риска как предиктор развития венозных тромбоэмболических осложнений / В. Е. Баринов, В. В. Бояринцев, Е. И. Брехов [и др.] // Клин. вест. хирург. 2013. - № 3. - С. 115-120.

3. Кириенко А. И. Острый тромбофлебит / А. И. Кириенко, А. А. Матюшенко, В. В. Андрияшкин. - М. : Литтерра, 2006. - 108 c.

4. Распространенность факторов риска венозного тромбоза у хирургических пациентов с тромботическими эпизодами в анамнезе / И. А. Санец, В. В. Аничкин, Н. И. Шевченко [и др.] // Проблемы здоровья и экологии. - 2015. - № 1 (43). C. 21-26. в сложном длительном высокостоимостном, часто пожизненным лечении [16].

Таким образом, несмотря на представленный массив данных о распространенности и факторах риска венозного тромбоза, остается до сих пор не оцененной реальная картина взаимодействия факторов риска, не проведена окончательная стратификация риска в первую очередь для ТГВ. Важными являются данные о сезонном тренде развития тромбоэмболий. Изучение распространенности, особенностей патогенеза тромботических осложнений у пациентов в послеоперационном периоде, совершенствование комплекса профилактических мероприятий этого вида послеоперационных осложнений имеет важное медико-социальное значение.

Выводы. По данным проведенного обзора установлено, что:

1. Свыше 70 \% тромбозов глубоких вен нижних конечностей после общехирургических операций протекают бессимптомно, но фатальная тромбоэмболия легочной артерии часто является первым и единственным проявлением венозного тромбоза, занимая третье место в общей структуре причин внезапной смерти.

2. Венозные тромбоэмболические осложнения сопровождаются поверхностным тромбофлебитом у 25 \% больных, при этом в большинстве случаев диагностируется тромбоз глубоких вен, а в 3,9 \% случае возникает тромбоэмболия легочной артерии.

3. Среди факторов хирургического риска венозных тромбоэмболий отмечена связь с общей анестезией, длительностью операции более 2 часов, тромбоцитозом, наличием асцита, хронической сердечной недостаточности, гипоальбуминемией.

5. Савельев В. С. Эндоваскулярная хирургия в профилактике тромбоэмболий легочной артерии и лечении острых венозных тромбозов / В. С. Савельев, В. И. Прокубовский, С. А. Капранов // Хирургия. - 2003. - № 2. - С. 6-11.

6. Седов В. М. Факторы риска тромботических осложнений при лапароскопических операциях и их профилактика / В. М. Седов, А. Б. Салов // Вестник хирургии им. И. И. Грекова. - 2003. - Т. 163, № 3. - С. 11-13.

7. Стрекаловский В. П. Профилактика тромбоэмболических осложнений при лапароскопической холецистэктомии / В. П. Стрекаловский, Ю. Г. Старков, К. В. Шишин // Хирургия. - 2004. - № 2. - С. 48-52.

8. Сухарев И. И. Лечение острой и хронической венозной недостаточности нижних конечностей с применением геля Лиотон - 1000 / И. И. Сухарев, Г. Г. Влайков // Клін. хірургія. - 1999. - № 6. - С. 5-6.

9. Чернуха Л. М. Клічні рекомендації з діагностики та лі- 
кування хронічних захворювань вен / Л. М. Чернуха // Клін. флебологія. - 2008. - № 1. - С. 27.

10. Clement D. L. Superficial vein thrombosis: more dangerous than anticipated / D. L. Clement // Phlebolymphology. - 2013. Vol 20, No. 4. - P. 188-192.

11. Cushman M. Epidemiology and risk factors for venous thrombosis / M. Cushman // Semin. Hematol. - 2007. - Vol. 44 (2). - P. 62-69.

12. Duration and magnitude of the postoperative risk of venous thromboembolism in middle aged women: prospective cohort study / S. Sweetland, J. Green, B. Liu [et al.] // B.M.J. - 2009. Vol. 339. - P. b4583.

13. Frequent asymptomatic pulmonary embolism in patients with deep venous thrombosis / K. M. Moser, P. F. Fedullo, J. K. Littejohn [et al.] // J. A. M. A. - 1994. - Vol. 271. - P. 223225.

14. Gillet J. L. Management of superficial vein thrombosis of the lower limbs: update and current recommendations / J. L. Gillet // Phlebolymphology. - 2015. - Vol. 22, No. 2. - P. 82-88.

15. Heit J. A. The epidemiology of venous thromboembolism in the community / J. A. Heit / Thromb. Haemost. - 2001. - Vol. 86, № 1. - P. 452-463

16. Henke P. K. Venous thromboembolism risk factor assessment and prophylaxis / P. K. Henke, C. J. Pannucci // Phlebology. 2010. - Vol. 25. - P. 219-223.

17. Identifying patients at high risk for venous thromboembolism requiring treatment after outpatient surgery / Ch. J. Pannucci, A. Shanks, M. J. Moote [et al.] // Ann. Surg. - 2012. - Vol. 255 (6). - P. 1093-1099.

18. Incidence and mortality of venous thrombosis: a populationbased study / I. A. Naess, S. C. Christiansen, P. Romundstad [et al.] // J. Thromb. Haemost. - 2007. - Vol. 5, № 4. - P. 692-699. 19. Incidence of symptomatic venous thromboembolism after different elective or urgent surgical procedures / R. H. White, H. Zhou, P. S. Romano // Thromb. Haemost. - 2003. - Vol. 90(3). - P. 446-455.

20. Incidence of venous thromboembolism in hospitalized patients vs community residents / J. A. Heit, L. J. Melton, C. M. Lohse [et al.] // Mayo Clinic Proceedings. - 2001. - Vol. 76, № 11. P. 1102-1110.

21. Incidence of venous thromboembolism in patients with cancer - a cohort study using linked United Kingdom databases / A. J. Walker, T. R. Card, J. West [et al.] // Eur. J. Cancer. - 2013. - Vol. 49 (6). - P. 1404-1413.

22. Kazaure S. Association of Postdischarge Complications With Reoperation and Mortality in General Surgery Hadiza / S. Kazaure, Sanziana A. Roman, Julie A. Sosa // Arch. Surg. 2012. - Vol. 147 (11). - P. 1001-1007.

23. Lijfering W. M. Risk factors for venous thrombosis current understanding from an epidemiological point of view / W. M. Lijfering, F. R. Rosendaal, S. Cannegieter // British Journal of Haematology. - 2010. - Vol. 149. - P. 824-833

24. Lowe G. D. O. Management of deep vein thrombosis to reduce the incidence of post-thrombotic syndrome / G. D. O. Lowe // Phlebology. - 2010. - Vol. 25. - P. 9-13.

25. Post-discharge venous thromboembolism after cancer surgery: extending the case for extended prophylaxis / R. P. Merkow, K. Y.
Bilimoria, M. E. Cohen [et al.] // Ann. Surg. - 2011. - Vol. 254 (1). - P. 131-137.

26. Post-discharge venous thromboembolism and associated mortality in general surgery: a population-based cohort study using linked hospital and primary care data in England / G. Bouras, E. M. Burns, A. M. Howell [et al.] // PLoS One. - 2015. Vol. 10 (12). - P. e0145759.

27. Poulikidis K. P. Prospective analysis of incidence, extent and chronicity of lower extremity venous thrombosis / K. P. Poulikidis, A. P. Gasparis, N. Labropoulos // Phlebology. 2014. - Vol. 29. - P. 37-42.

28. Prevention of venous thromboembolism in major orthopedic surgery / P. Prandoni, S. Z. Goldhaber, A. Piccioli [et al.] // Clin. Appl. Thromb. Hemost. - 1996. - Vol. 3. - P. 153-157

29. Pulmonary embolism: Epidemiology and registries / M. Monreal, I. Mahé, A. Bura-Riviere [et al.] // Presse Med. 2015. - Vol. 44. - P. 377-383.

30. Risk factors and clinical impact of postoperative symptomatic venous thromboembolism / C. Gangireddy, J. R. Rectenwald, G. R. Upchurch [et al.] // J. Vasc. Surg. - 2007. - Vol. 45 (2). P. 335-341.

31. Risk factors and short-term mortality of venous thromboembolism diagnosed in the primary care setting in the United Kingdom / C. Huerta, S. Johansson, M. A. Wallander [et al.] // Arch. Intern. Med. - 2007. - Vol. 167 (9). - P. 935-943.

32. Superficial venous thrombosis and venous thromboembolism. A large, prospective epidemiologic study / H. Decousus, I. Quéré, E. Presles [et al.] // Ann. Intern. Med. - 2010. - Vol. 152, No. 4. - P. 218-224.

33. Swiet M. Risk of and prophylaxis for venous thromboembolism in hospital patients. Thromboembolic Risk Factors (THRIFT) Consensus Group / M. Swiet // B. M. J. - 1992. - Vol. 305 (6853). - P. 567-574.

34. The incidence of post operative venous thromboembolism in patients undergoing varicose vein surgery recorded in Hospital Episode Statistics / P. A. Sutton, Y. El-Duhwaib, J. Dyer [et al.] // Ann. R. Coll. Surg. Engl. - 2012. - Vol. 94 (7). - P. 481-483. 35. The results of the surgical treatment of superficial venous thrombosis. Cercetari Experimentale \& Medico-Chirurgicale / J. Avram, F. Cadariu, M. Pasztori [et al.] // J. Experim. Med. Res. - 2010. - Vol. 17, No. 3. - P. 79-84.

36. Upper-extremity deep vein thrombosis. Risk factors, diagnosis, and complications / P. Prandoni, P. Polistena, E. Bernardi [et al.] // Arch. Intern. Med. - 1997. - Vol. 157. - P. 57-62.

37. Venous thromboembolism: reducing the risk of venous thromboembolism (deep vein thrombosis and pulmonary embolism) in patients admitted to hospital Produced by the National Clinical Guideline Centre - Acute and Chronic Conditions (formerly the National Collaborating Centre for Acute Care) / T. Treasure, K. Carter, N. Gautam [et al.]. - 2015, 2nd ed. - 519 p.

38. VTE Impact Assessment Group in Europe (VITAE). Venous thromboembolism (VTE) in Europe. The number of VTE events and associated morbidity and mortality / A. T. Cohen, G. Agnelli, F. A. Anderson [et al.] // Thromb. Haemost. - 2007. - Vol. 98. - P. 756-764.

39. White R. H. The Epidemiology of venous thromboembolism / R. H. White // Circulation. - 2003. - Vol. 107. - P. 4-8. 


\section{REFERENCES}

1. Zatevakhin, I.I. (2001). Rol ultrazvukovogo angioskanirovaniya $v$ rannyey diagnostike bezsymptomnykh ostrykh venoznykh trombozov sistemy nizhney polovoy veny u operyrovannykh bolnykh [The role of ultrasound angioscanning in the early diagnosis of asymptomatic acute venous thrombosis of the inferior vena cava system in operated patients]. Materialy III konferentsyy assotsyatsyy flebologov Rossii - Materials of the III Conference of the Russian Association of Phlebologists. Rostov-naDonu, [in Russian].

2. Barynov, V.E., Boyaryntsev, V.V. \& Brekhov, E.I. (2013). Integral faktorov riska kak prediktor razvitiya venoznykh tromboembolycheskikh oslozhneniy [The integral of risk factors as a predictor of the development of venous thromboembolic complications]. Klin. Vest. Khir. - Clinical Journal of Surgery, 3, 115120 [in Russia].

3. Kiriyenko, A.I., Matyushenko, A.A. \& Andriyashkin, V.V. (2006). Ostryy tromboflebit [Acute thrombophlebitis]. Moscow: Litterra [in Russian].

4. Sanyets, I.A., Anichkin, V.V. \& Shevchenko, N.I. (2015). Rasprostranonnost faktorov riska venoznogo tromboza u khirurhicheskikh patsientov s tromboticheskimy epizodami v anamneze [Prevalence of risk factors for venous thrombosis in surgical patients with history of thrombotic episodes]. Problemy zdorovya i ekologii - Problems of Health and Ecology, 1 (43), 21-26 [in Russian].

5. Savelyev, V.S., Prokubovskiy, V.I. \& Kapranov, S.A. (2003). Endovaskulyarnaya khirurgiya v profilaktike tromboemboliy legochnoy arterii i lechenii ostrykh venoznykh trombozov [Endovascular surgery in the prevention of pulmonary embolism and treatment of acute venous thrombosis]. Khirurgiya - Surgery, 2, 6-11 [in Russian].

6. Sedov, V.M. \& Salov. A.B. (2003). Faktory riska tromboticheskikh oslozhnyeniy pry laparoskopicheskikh operatsiyakh i ikh profilaktika [Risk factors for thrombotic complications in laparoscopic operations and their prevention]. Vestnik khirurgii im. I.I. Grekova - Journal of Surgery named after I.I. Grekov, 163 (3), 11-13 [in Russian].

7. Strekalovskyy, V.P., Starkov, Yu.G. \& Shyshyn, K.V. (2014). Profilaktika tromboembolicheskikh oslozhneniy pry laparoskopicheskoy kholetsystektomii [Prevention of thromboembolic complications in laparoscopic cholecystectomy]. Khirurgiya - Surgery, 2, 48-52 [in Russian].

8. Sukharev, I.I. \& Vlaykov, G.G. (1999). Lecheniye ostroy i khronicheskoy venoznoy nedostatochnosti nizhnikh konechnostey s primeneniyem gelya Lioton-1000 [Treatment of acute and chronic venous insufficiency of the lower extremities with the use of Lyoton gel-1000]. Klin. Khirurgiya - Clinical Surgery, 6, 5-6 [in Russian].

9. Chernukha, L.M. (2008). Klinichni rekomendatsii z diahnostyky ta likuvannia khronichnykh zakhvoriuvan ven [Clinical guidelines for the diagnosis and treatment of chronic venous disease]. Klin. Flebolohiia - Clinical Phlebology, 1, 27 [in Ukrainian].

10. Clement, D.L. (2013). Superficial vein thrombosis: more dangerous than anticipated. Phlebolymphology, 20 (4), 188-192.

11. Cushman, M. (2007). Epidemiology and risk factors for venous thrombosis. Semin. Hematol., 44 (2), 62-69.

12. Sweetland, S., Green, J., \& Liu, B. (2009). Duration and magnitude of the postoperative risk of venous thromboembolism in middle aged women: prospective cohort study. B.M.J., 339, b4583.

13. Moser, K.M., Fedullo, P.F. \& Littejohn, J.K. (1994). Frequent asymptomatic pulmonary embolism in patients with deep venous thrombosis. J. A.M.A. 271, 223-225.

14. Gillet, J.L. (2015). Management of superficial vein thrombosis of the lower limbs: update and current recommendations. Phlebolymphology, 22 (2), 82-88.

15. Heit, J.A. (2001). The epidemiology of venous thromboembolism in the community. Thromb. Haemost., 86 (1), 452-463. 16. Henke,P.K. \& PannucciC.J.(2010). Venous thromboembolism risk factor assessment and prophylaxis. Phlebology, 25, 219-223. 17. Pannucci, Ch.J. Shanks, A. \& Moote M.J. (2012). Identifying patients at high risk for venous thromboembolism requiring treatment after outpatient surgery. Ann. Surg., 255 (6), 1093-1099. 18. Naess, I.A., Christiansen, S.C. \& Romundstad P. (2007). Incidence and mortality of venous thrombosis: a population-based study. J. Thromb. Haemost., 5, (4), 692-699.

19. White, R.H., Zhou, H. \& Romano, P.S. (2003). Incidence of symptomatic venous thromboembolism after different elective or urgent surgical procedures. Thromb. Haemost., 90 (3), 446-455.

20. Heit, J.A., Melton, L.J. \& Lohse C.M. (2001). Incidence of venous thromboembolism in hospitalized patients vs community residents. Mayo Clinic Proceedings, 76 (11), 1102-1110.

21. Walker, A.J., Card, T.R. \& West J. (2013). Incidence of venous thromboembolism in patients with cancer - a cohort study using linked United Kingdom databases. Eur. J. Cancer., 49 (6), 1404-1413.

22. Kazaure, S., Sanziana, A., Roman, \& Julie, A. Sosa (2012). Association of post-discharge complications with reoperation and mortality in General Surgery Hadiza. Arch. Surg., 147 (11), 10011007.

23. Lijfering, W.M., Rosendaal, F.R. \& Cannegieter S. (2010). Risk factors for venous thrombosis - current understanding from an epidemiological point of view. British Journal of Haematology, 149, 824-833.

24. Lowe, G.D.O. (2010). Management of deep vein thrombosis to reduce the incidence of post-thrombotic syndrome. Phlebology, 25, 9-13.

25. Merkow, R.P., Bilimoria, K.Y. \& Cohen, M.E. (2011). Post-discharge venous thromboembolism after cancer surgery: extending the case for extended prophylaxis. Ann Surg., 254 (1), 131-137.

26. Bouras, G., Burns, E.M. \& Howell, A.M. (2015). Postdischarge venous thromboembolism and associated mortality in General surgery: a population-based cohort study using linked hospital and primary care data in England. PLoS One, 10, 12, e0145759.

27. Poulikidis, K.P., Gasparis, A.P. \& Labropoulos N. (2014). Prospective analysis of incidence, extent and chronicity of lower extremity venous thrombosis. Phlebology, 29, 37-42.

28. Prandoni, P., Goldhaber, S.Z. \& Piccioli A. (1996). Prevention of venous thromboembolism in major orthopedic surgery. Clin. Appl. Thromb. Hemost., 3, 153-157.

29. Monreal, M., Mahé, I. \& Bura-Riviere, A. (2015). Pulmonary embolism: Epidemiology and registries. Presse Med., 44, 377383.

30. Gangireddy, C., Rectenwald, J.R. \& Upchurch G.R. (2007). Risk factors and clinical impact of postoperative symptomatic venous thromboembolism. J. Vasc. Surg., 45 (2), 335-341.

31. Huerta, C., Johansson, S. \& Wallander, M.A. (2007). Risk factors and short-term mortality of venous thromboembolism diagnosed in the primary care setting in the United Kingdom. Arch. Intern. Med., 167 (9), 935-943.

32. Decousus, H., Quéré, E. \& Presles, I. (2010). Superficial venous thrombosis and venous thromboembolism. A large, prospective epidemiologic study. Ann. Intern. Med., 152 (4), 218-224. 
33. Swiet, M. (1992). Risk of and prophylaxis for venous thromboembolism in hospital patients. Thromboembolic Risk Factors (THRIFT) Consensus Group. B.M.J., 305 (6853), 567574.

34. Sutton, P.A., El-Duhwaib, Y. \& Dyer J. (2012). The incidence of post operative venous thromboembolism in patients undergoing varicose vein surgery recorded in Hospital Episode Statistics. Ann. R. Coll. Surg. Engl., 94 (7), 481-483.

35. Avram, J., Cadariu, F. \& Pasztori, M. (2010). The results of the surgical treatment of superficial venous thrombosis. Cercetari Experimentale \& Medico-Chirurgicale. J. Experim. Med. Res., 17 (3), 79-84.

36. Prandoni, P., Polistena, P., \& Bernardi, E. (1997). Upperextremity deep vein thrombosis. Risk factors, diagnosis, and complications. Arch. Intern. Med., 157, 57-62.

37. Treasure, T., Carter, K. \& Gautam, N. ( $2^{\text {nd }}$ ed.) (2015). Venous thromboembolism: reducing the risk of venous thromboembolism (deep vein thrombosis and pulmonary embolism) in patients admitted to hospital Produced by the National Clinical Guideline Centre - Acute and Chronic Conditions (formerly the National Collaborating Centre for Acute Care), $519 \mathrm{p}$.

38. Cohen, A.T., Agnelli, G. \& Anderson, F.A. (2007). VTE Impact Assessment Group in Europe (VITAE). Venous thromboembolism (VTE) in Europe. The number of VTE events and associated morbidity and mortality. Thromb. Haemost., 98, 756-764.

39. White, R.H. (2003). The Epidemiology of venous thromboembolism. Circulation, 107, 4-8.

\title{
YAN SIAO
}

P. Shupyk National Medical Academy of Postgraduate Education

\section{INCIDENCE OF VENOUS THROMBOSIS IN EUROPEAN POPULATIONS: ROLE OF SURGERY}

\begin{abstract}
The article discusses the incidence and role of surgical interventions in the development of venous thrombosis. According to the survey it was found that more than $70.0 \%$ of deep vein thrombosis of lower limbs after general surgical operations occur asymptomatically, while fatal pulmonary artery thromboembolism is often the first and only manifestation of venous thrombosis, occupying the third place in the overall structure of the causes of sudden death. Venous thromboembolic complications are accompanied by superficial thrombophlebitis in $25.0 \%$ of patients, with most cases diagnosed deep vein thrombosis, and $3.9 \%$ in case of pulmonary embolism occurs. Surgical risk factors of venous thromboembolism have marked links with general anesthesia, operation duration over 2 hours, thrombocytosis, the presence of ascites, chronic heart failure and the presence of hypoalbuminemia.
\end{abstract}

Key words: vein thrombosis; pulmonary thromboembolism; incidence; surgical interventions.

\section{ЯН СЯО}

Національна медична академія післядипломної освіти імені П. Л. Шупика

\section{ІНЦИДЕНТНІСТЬ ВЕНОЗНИХ ТРОМБОЗІВ В ЄВРОПЕЙСБКІЙ ПОПУЛЯЦЇ: РОЛЬ ХІРУРГЧНИХ ВТРУЧАНЬ}

У статті розглянуто інцидентність і роль хірургічних втручань у розвитку венозних тромбозів. За даними проведеного огляду встановлено, що понад 70 \% тромбозів глибоких вен нижніх кінцівок після загальнохірургічних операцій перебігають безсимптомно, при цьому фатальна тромбоемболія легеневої артерії часто є першим і єдиним проявом венозного тромбозу, посідаючи третє місце в загальній структурі причин раптової смерті. Венозні тромбоемболічні ускладнення супроводжуються поверхневим тромбофлебітом у 25 \% хворих, при цьому в більшості випадків діагностується тромбоз глибоких вен, а в 3,9 \% випадків виникає тромбоемболія легеневої артерії. Серед чинників хірургічного ризику венозних тромбоемболій спостерігається зв'язок із загальною анестезією, тривалістю операції більше 2 год, тромбоцитозом, наявністю асциту, хронічної серцевої недостатності, гіпоальбумінемією.

Ключові слова: венозний тромбоз; інцидентність; хірургічні втручання. 